\title{
e-Phaïstos
}

e-Phaïstos

Revue d'histoire des techniques / Journal of the history

of technology

IV-1 | 2015

Les arts de guerre et de grâce (XIVe-XVIIle siècles)

\section{Entretien avec Paul Benoit. Les relations entre l'archéologie et l'histoire des techniques}

Par Ivan Lafarge

Interview with Paul Benoit by Ivan Lafarge. Links between the archeology and the history of the technology

\section{Paul Benoit et Ivan Lafarge}

\section{(2) OpenEdition}

Journals

\section{Édition électronique}

URL : https://journals.openedition.org/ephaistos/834

DOI : 10.4000/ephaistos.834

ISSN : 2552-0741

Éditeur

IHMC - Institut d'histoire moderne et contemporaine (UMR 8066)

Édition imprimée

Date de publication : 1 avril 2015

Pagination : 84-96

ISSN : 2262-7340

Référence électronique

Paul Benoit et Ivan Lafarge, «Entretien avec Paul Benoit. Les relations entre l'archéologie et l'histoire des techniques », e-Phaïstos [En ligne], IV-1 | 2015, mis en ligne le 06 décembre 2016, consulté le 19 février 2023. URL : http://journals.openedition.org/ephaistos/834 ; DOI : https://doi.org/10.4000/ ephaistos.834 


\section{Entretien avec Paul Benoit Les relations entre l'archéologie et l'histoire des techniques}

\begin{abstract}
Cursus académique
Professeur émérite de l'Université Paris 1, Paul Benoit a mis en place et dirigé l'équipe " Histoire des Techniques » de $1975^{1}$ à 2005, date à laquelle il a été remplacé par Anne-Françoise Garçon. D'abord rattachée au Lamop, cette équipe est aujourd'hui rattachée à l'IHMC, UMR8066 CNRS.

Il a beaucoup travaillé sur l'histoire et l'archéologie des mines et de la métallurgie (fouilles de Pampailly et de l'abbaye de Fontenay), mais aussi sur la construction et les carrières, l'hydraulique, les moulins, l'urbanisme et de nombreux sujets connexes.
\end{abstract}

\section{Bibliographie sélective}

Avec P. Braunstein : Mines, carrières et métallurgie dans la France médiévale, CNRS, 1983.

"Technology and crisis: The great depression of the Middle Ages and the technology of Renaissance (XIVth to XVIth centuries) », dans History and Technology, 1984, vol.1, 319-333.

Avec D. Cailleaux: Hommes et travail du métal dans les villes médiévales, actes de la table ronde réunie à Paris le 23 mars 1984, éditions AEDEH, diffusion Picard, Paris, 1988, 254 p.

Avec D. Cailleaux: Moines et métallurgie dans la France médiévale, Association pour l'édition et la diffusion des études historiques, Paris 1991.

Avec A. Bideau, Mines et métallurgie, Villeurbanne, Programme Rhône-Alpes recherches en sciences humaines, 1994.

Avec P. Fluzin, Paléométallurgie du fer \& cul-

\section{Par Ivan Lafarge}

tures : actes du Symposium international du Comité pour la sidérurgie ancienne de l'Union internationale des sciences préhistoriques et protohistoriques, Belfort ; Paris, Vulcain ; AEDEH, 1995.

Avec Léon Pressouyre, L'hydraulique monastique, milieux, réseaux, usages, Rencontres à Royaumont, éditions Créaphis, 1996, 516 p.

La mine de Pampailly (XVe-XVIIIe siècles), Brussieu, Rhône, Lyon, Service régional de l'archéologie, 1997 Documents d'archéologie en RhôneAlpes, 14, $137 \mathrm{p}$.

Avec J. Lorenz et D. Obert, Pierres \& carrières : géologie, archéologie, histoire : textes réunis en hommage à Claude Lorenz : actes des journées Claude Lorenz, Paris 17 et 18 novembre 1995, Paris, A.E.D.E.H, 1997. Partiellement Édité en ligne : http://www.lrmh.culture ... 03D3626746368703D7 o726E

Avec C. Verna, Le charbon de terre en Europe occidentale avant l'usage industriel du coke, Proceedings of the XXth International Congress of History of Sciences (Liège, 20-26 July 1997), Turnhout, Brépols 1999, p.41-48.

Avec P. Pétrequin, P. Fluzin et J. Thiriot, Arts $d u$ feu et productions artisanales, Actes des XXe Rencontres Internationales d'Archéologie et d'Histoire d'Antibes, 21-23octobre 1999, éditions APDCA, Antibes, 2000.

Avec K. Berthier Agriculture et aménagement du paysage hydrologique dans le bassin de la Seine aux XIVe - XVe siècle, Colloque du Programme Environnement, Vie et Sociétés, CNRS, Paris, 25 juin, 2003.

Avec O. Mattéoni, Pêche et pisciculture en eau 
douce : la rivière et l'étang au Moyen Âge. Actes des 1ères rencontres internationales de Liessies, Lille, Conseil Général du Nord, 2004, publication CD-Rom.

Avec K. Berthier et J. Rouillard éd., L'industrie cistercienne, Cannes, Institut coopératif de l'école moderne (France), 2004 (Bibliothèque de travail)

Avec J. Rouillard, Analyse historique du peuplement de la Seine, PIREN Seine, rapport 2006, CNRS UMR 7619 Sisyphe, Paris, 2006.

\section{Entretien}

e-Phaïstos : Peut-on faire de l'histoire en prenant comme source les objets matériels, je pense notamment à Antoine Prost qui expliquait récemment son intérêt en tant qu'historien pour les objets matériels qui sont de véritables sources historiques $^{2}$; qu'est-ce qui différencie l'archéologie et l'histoire?

Paul Benoit : Oui bien sûr! D'ailleurs l'étude des deux conflits mondiaux illustre bien cette question. En ce qui concerne la première guerre mondiale, l'intérêt de l'étude archéologique de ses vestiges n'est plus à démontrer ${ }^{3}$, alors même que parfois ces fouilles posent de gros problèmes de sécurité. Quant au rapport entre archéologie et histoire, je ne ressens pas de différence fondamentale entre les deux approches. S'il y a une différence, elle est très minime et d'ordre théorique, l'objet de l'une comme l'autre discipline est la science historique ; une approche raisonnée du passé, et moi je suis autant historien qu'archéologue.

Pour certains comme moi, la démarche au départ est celle d'un historien, pour d'autres, je pense à quelqu'un comme Marie-Christine Bailly-Maitre4 par exemple, le point de départ est l'archéologie. On finit par se retrouver et on a travaillé suffisamment ensemble pour être sur la même longueur d'onde.

e-Phaïstos : Comment s'imbriquent les questions qu'on va poser à partir des documents d'archives avec des problématiques d'ordre archéolo- gique, au-delà de la simple question de comment fonctionne la forge ? Ou est-ce que ce qui fait l'histoire des techniques c'est tout simplement la question du fonctionnement de la forge ? Comment s'organise cette recherche et les passerelles d'une discipline à l'autre?

P.B. : Je dirais que toute démarche scientifique nécessite une certaine forme de curiosité, sans curiosité, il n'y a pas de science, si on veut comprendre ce qu'est une forge il faut automatiquement une curiosité qui va loin puisqu'elle vise à comprendre le passé, et comment elle s'organise... Pour moi c'est venu tout seul, c'est tellement évident que je ne me suis pas posé le problème de comment j'étais passé de l'histoire à l'archéologie. A partir du moment où je me suis posé la question de savoir ce qu'était une forge, ce qu'était un moulin, combien il y en avait, il m'a fallu plonger le nez dans l'archéologie. Mais restaient des questions que l'archéologie ne pouvait pas traiter, c'est-à-dire à qui ils appartenaient, qui y travaillait (ce qui est bien difficile à savoir), à qui cela profitait. Il y a donc toute une série de données à caractère pour moi économique. Je fais de l'archéologie des techniques et de l'archéologie de la production, quelqu'un qui fait de l'archéologie du sacré se posera d'autres problèmes. On va retrouver aussi le rapport avec le texte à un moment ou à un autre; là ce sont des choses qui vont tellement de soi que j'ai beaucoup de mal à répondre. Mon point de départ n'est pas une position théorique sur ce que doit être l'histoire et l'archéologie, c'est ma pratique de ce que j'appellerai l'histoire des techniques qui m'a conduit à l'archéologie.

e-Phaïstos : Souvent l'archéologie universitaire est une archéologie programmée ; qu'est-ce que le développement de l'archéologie préventive a permis d'apporter ou générer comme contrainte vis-à-vis de ce type de recherche?

P.B. : C'est un vaste problème... Je suis en plein dedans parce que je fais la préface d'un livre écrit par Marc Leroy 5 sur la sidérurgie médiévale en Lor- 
raine. Il part d'un questionnement très scientifique au sens étroit du terme : ce qu'il veut savoir c'est si la minette lorraine, ce minerai qu'on a extrait par millions de tonnes et dont il reste encore des millions de tonnes dans le sous-sol lorrain, a servi ou non à l'époque médiévale. Ensuite il se pose la question de savoir si on s'en est servi un peu ou beaucoup, il arrive à cette conclusion au terme d'un très beau et très gros travail que la minette lorraine a fourni l'essentiel du fer médiéval... en Lorraine. A cette époque, on fait très peu circuler le minerai, en tous cas pas sur des centaines de kilomètres...

La Lorraine a donc produit son fer en utilisant la minette, c'est-à-dire un minerai phosphoreux, considéré comme trop phosphoreux par les métallurgistes des XVIII et XIX ${ }^{\mathrm{e}}$ siècles qui travaillaient en réduction indirecte. C'est là qu'on retrouve un jeu entre histoire et archéologie : les gens qui avaient posé comme principe qu'on ne pouvait pas utiliser la minette avant l'invention du procédé de Thomas Gilchrist ${ }^{6}$, pensaient de la minette « ce fer est trop phosphoreux pour être de qualité ». Marc Leroy démontre le contraire. Ce qui est intéressant dans son cas, c'est qu'il a fait aussi bien des fouilles préventives que programmées, il a commencé avec des contrats AFAN7 en fouille urbaine alors que l'INRAP n'existait pas encore. Il a fait quelques fouilles préventives et un travail énorme en programmée. L'avantage du préventif c'est que ça donne des moyens matériels, en hommes et en argent, extrêmement importants par rapport à ceux des fouilles programmées ${ }^{8}$. Alors si on travaille sur l'histoire du fer c'est extrêmement intéressant parce que on va pouvoir décaper le long des autoroutes des surfaces considérables et lorsqu'on on tombe sur une zone d'extraction minière, c'est le paradis. C'est actuellement le cas dans l'ouest de la France, dans la Sarthe ${ }^{9}$ avec des chantiers autoroutiers et TGV énormes. Donc l'archéologie préventive a permis de découvrir des quantités très importantes de sites qui jusque-là passaient complètement inaperçus. Là, on se rend compte que Rolf Sprandel, qui disait de façon intuitive qu'à l'époque médiévale, c'est probablement la France qui avait produit le plus de fer $^{10}$, il avait sans doute raison, or ce n'était que sa réflexion qui' l'avait conduit à cette conclusion. On a beaucoup moins de chance avec les moulins puisqu'il est très rare que les autoroutes suivent le cours des petites rivières. Il y a des exceptions quand même, des heureuses exceptions, comme c'est le cas du moulin de Thervay ${ }^{11}$ que je connais bien, puisque j'y ai un peu fouillé, en fait j'étais expert sur ce site. Une très belle fouille menée pour l'INRAP, par Gilles Rollier. On pouvait enfin fouiller un moulin. Il fallait de gros moyens à cause de l'eau, du temps, du personnel... On aurait eu beaucoup de mal à fouiller ça en programmée.

Par contre il y a certaines recherches qu'on ne fait en général qu'en programmée, comme les prospections thématiques : j'en reviens au cas de mon ami Marc Leroy, il a fait des prospections forestières extrêmement importantes dans des zones où ne passeront jamais, au grand jamais, ni le TGV, ni l'autoroute, il a fait des kilomètres et des kilomètres à pied à la recherche de vestiges de réduction du fer ou de vestiges de puits de mine; on l'a également fait dans la région de Fontenay.

La thèse récente encore de Christophe Colliou ${ }^{12} \mathrm{a}$ montré tout ce qu'on pouvait tirer de ce qu'on voyait du ciel, généralement la prospection aérienne se fait en programmée.

Il y a donc pour moi une complémentarité à établir, entre des programmes à mener de manière complémentaire avec les fouilles préventives dont le très gros inconvénient est généralement le manque de temps et surtout la contrainte spatiale liée aux surfaces d'aménagement. J'étais sur un chantier au nord de Sully-sur-Loire pour un bout d'autoroute, le décapage avait dégagé une moitié d'atelier et il était impossible de fouiller la seconde moitié située hors emprise de l'aménagement. Cette contrainte est très frustrante.

Des choses comme ce qui s'est fait en France en archéologie minière ne sont concevables qu'en archéologie programmée parce que c'est exceptionnel qu'on arrive à couper une galerie de mine lors de 
grands travaux. Cela a pu se produire, mais c'est extrêmement rare et ça ne permet pas d'explorer la mine (ça reste un échantillonnage). J'avais été sollicité par Philippe Nowacki ${ }^{13}$, un type remarquable, pour travailler en préventif sur une mine en Guyane lors des fouilles préventives du barrage de Petit Saut, mais il est décédé et son successeur a fait appel à quelqu'un d'autre, je le regrette beaucoup.

e-Phaïstos : Ne faut-il pas revoir un certain nombre de résultats de l'archéologie préventive à l'aune de la programmation scientifique, en les requestionnant par rapport à des problématiques de recherches en cours?

P.B. : On a tendance à vouloir opposer les archéologues préventifs et programmés, je crois qu'il y a plus de conflits parmi les gens qui font du préventif, qu'entre programmé et préventif. Malheureusement dans certains cas on se demande pourquoi des fouilles ont eu lieu, les résultats ne sont pas publiés, ou si mal... Un exemple qui me semble très très intéressant et significatif à la fois de la bonne volonté des gens qui font du préventif et du manque de moyens alloués à la publication. J'ai fouillé à l'abbaye de Fontmorigny des caissons à poisson, dans un vivier, dans lesquels on met les poissons, généralement les carnassiers pour qu'ils ne dévorent pas les petits et qu'on puisse les attraper plus facilement ${ }^{14}$. C'était une fouille programmée. J'ai appris par un dendrochronologue qu'une collègue en avait fouillé un avec l'AFAN en $\mathrm{Au}$ vergne et n'avait pas pu le publier, renseignements pris, elle m'a envoyé son dossier et j'ai pu le passer avec mes affaires, si ce n'est qu'il manquait des éléments parce qu'elle n'avait pas eu assez d'argent pour affiner fouille et analyse. Cependant je ne peux qu'en juger assez mal parce que les gens que je fréquente et qui font du préventif sont des gens qui travaillent plus ou moins en relation avec moi et sont des gens pour qui l'archéologie c'est aussi la publication.

Parmi ce qui se fait difficilement en préventif, je pense qu'on pourrait aussi prendre l'exemple des carrières et tout ce qui est souterrain. Même si depuis quelques années des carrières ont pu être documentées à défaut d'être complètement fouillées par le préventif. Mais toutes les vraies fouilles ont été faites en programmée, le travail sur les carrières ça ne rentre généralement pas dans les cases, car c'est quand même assez particulier.

e-Phaïstos : Oui, c'est vrai mais Marc Viré ${ }^{5}$, fait là-dessus la réflexion suivante : la carrière génère des travaux de dégagement si importants et chers pour un résultat si peu visible, à tout le moins pour les non spécialistes que ces fouilles sont très rarement prescrites.

P.B. : Oui, la mine c'est pareil. Les gens qui font du préventif sont là pour sauver les vestiges et les étudier de manière à ce que la mémoire des vestiges qui sont détruits soit non parfaitement, mais conservée le mieux possible et c'est déjà énorme. Pour reprendre l'exemple du moulin de Thervay, on sait maintenant ce qu'était ce moulin, mais il n'en reste plus rien, il a été intégralement fouillé (donc détruit) et après on a creusé des fondations et coulé du béton pour soutenir le petit pont sur lequel passe le TGV. Mais ce n'est pas seulement le lot des fouilles préventives, c'est un des moyens de l'archéologie que de détruire ce qu'elle étudie. Dès lors qu'on fait des fouilles se posent des questions de choix de conservation.

Je m'intéresse aussi aux rapports entre histoire et archéologie et sciences dites exactes.

e-Phaïstos : J'avais pensé à une question sur le rapport entre histoire des techniques et histoire des sciences...

P.B. : Là je ne suis pas au niveau de l'histoire des sciences, je pensais aux développements actuels de méthodes scientifiques ou de découvertes techniques pour ce que ça apporte à l'archéologie et en particulier l'archéologie des techniques. Pour nous c'est absolument indispensable : si on veut savoir comment fonctionnait un four de réduction du minerai de fer, il faut des analyses, des analyses du 
minerai, des analyses des scories, des analyses de tous types. Avant de commencer à faire de la métallographie, maintenant on fait des analyses chimiques très poussées, avec étude non seulement des éléments majeurs, mais aussi des éléments mineurs, et des éléments traces, cela permet de repérer, parfois, la provenance du minerai. Là encore, le travail de Marc Leroy est exemplaire. A Fontenay on a pu démontrer des choses assez intéressantes : le métal qu'on y obtenait était tout-à-fait comparable à ce qui servait à la même époque (XII ${ }^{2}-\mathrm{XIII}^{\mathrm{e}}$ siècles) à fabriquer les tirants et autres pièces d'armature des cathédrales; c'est à peu près le même métal que les premiers canons. C'est-à-dire un fer assez impur.

Ce que peut apporter la métallographie est donc tout-à-fait intéressant, cela donne des possibilités d'établir vraiment une filière technique, de la voir non seulement d'un point de vue théorique, mais également dans la pratique. Cela donne à voir aussi les résultats, je dirais économiques et matériels de ce travail : comment on passe du minerai de fer à un boulet de canon dans certains cas (pour l'indirect) ou du minerai de fer à des tirants d'église. Ce sont les analyses qui nous ont montré par exemple qu'à la cathédrale de Beauvais, le cerclage est issu d'un minerai qui n'est pas le minerai local ; ça débouche sur l'histoire économique. Les antiquisants làdessus nous ont donné des leçons avec la chance qu'ils ont eu de retrouver des épaves en Méditerranée chargées de lingots de métal, que ce soit du plomb ou que ce soit de barres de fer. Les analyses nous apportent énormément dans ce domaine-là.

On a eu récemment un colloque international à Namur et Dinant sur les alliages cuivreux à l'époque médiévale ${ }^{16}$. On nous y a présenté énormément d'analyses qui apportent beaucoup de choses, mais ça ne résout pas tous les problèmes : il y a eu par exemple de grandes discussions sur la détermination des origines du minerai qui a donné certaines pièces trouvées en Angleterre par exemple : sontelles d'origine anglaise ou d'origine flamande? La question n'a pas vraiment été tranchée mais on commence à avoir des idées de réponse ${ }^{17}$.

Le second point concerne tous les problèmes liés à l'environnement: l'archéologie, liée cette fois-ci non pas à l'histoire, mais aux sciences de l'environnement, et en particulier aux gens qui travaillent sur l'histoire du climat, nous apprend énormément de choses. Les analyses physico-chimiques et la métallographie sont des questions développées depuis longtemps, il $\mathrm{y}$ a de très beaux exemples dès la fin du XIXe siècle où des gens commençaient à s'y intéresser ${ }^{18}$. L'histoire du climat et de la végétation est une recherche beaucoup plus actuelle, si le recueil des données concernant le climat a pu être, un peu, le travail d'historiens ${ }^{19}$, à mon avis c'est à partir d'analyses des éléments retrouvés et prélevés dans les glaciers, la calotte polaire ou autres qu'on peut renouveler les données paléoclimatologiques, la glaciologie permet de franchir un pas. Se posent aussi maintenant toutes les questions de géomorphologie, il y a notamment dans la sphère de l'archéologie des gens compétents là-dessus: problèmes d'érosion, de variation du lit des cours d'eau, etc. Ce qui est intéressant c'est qu'on est passés d'une étude partie des géographes vers les archéologues et qui intéresse maintenant les historiens. Ils travaillent à partir de cartes anciennes ou même de textes en croisant les données. C'est ce que fait Joséphine Rouillard en ce moment, elle reprend les données environnementales de la Bassée à l'époque médiévale à partir des travaux de Daniel Mordant et son équipe, ce sont des données issues de l'archéologie préventive entre les années 1960 et les années 2000, puisque ce sont des sauvetages de gravières. Alors elle reprend les textes médiévaux pour essayer de comprendre comment ça fonctionnait à l'époque médiévale parce que malheureusement, les grandes données archéologiques s'arrêtent à l'époque carolingienne ${ }^{20}$, quelques fermes au bas Moyen Age, mais les textes nous en apprennent plus.

L'intégration à la recherche historique et archéologique des sciences de la terre - sciences de la vie est un domaine actuellement en mouvement qui 
intéresse autant les historiens que les archéologues, et ce sont des domaines où actuellement il $\mathrm{y}$ a plus de moyens que les ressources habituelles de l'archéologie et de l'histoire. Ce sont des sujets de recherche qui sont aussi un peu à la mode et puis qui sont considérés comme indispensables maintenant. Actuellement on fait marcher l'équipe du Lamop ${ }^{21}$ en grande partie avec l'argent du programme interdisciplinaire sur l'environnement de la Seine (le Piren-Seine), c'est quelque chose d'assez neuf ; l'histoire des techniques peut être considérée maintenant comme quelque chose d'utile par des utilitaristes ; par des gens qui manient les cordons de la bourse.

Un certain nombre d'études sur la métallurgie médiévale ont été financées par des programmes industriels sur le vieillissement des métaux. Pourquoi s'intéresse-t-on tant au vieillissement du fer ? Parce que c'est un domaine d'anticipation industrielle : pour faire des conteneurs en plomb doublé d'acier qui contiendront, ou qui contiennent déjà des déchets nucléaires. Donc l'histoire de la métallurgie bénéficie en particulier à Compiègne ${ }^{22}$ avec Philippe Fluzin ${ }^{23}$ et Philippe Dillmann ${ }^{24}$, de contrats sur le vieillissement des métaux.

e-Phaïstos : Il y a actuellement une équipe de paléométallurgie au CNRS assez importante associée au CEA au sein de laquelle travaillent des archéologues médiévistes et protohistoriens (Sylvain Bauvais $^{25}$, Philippe Dillmann...).

P.B. : Oui ils sont venus sur le site de Danièle Arribet ${ }^{26}$, pour faire des mesures. Tout ça c'est un ensemble qui est de plus en plus complexe et qui prouve que l'équation histoire + archéologie $=$ quelque chose, on atteint maintenant des domaines scientifiques auxquels il faut que l'historien ou l'archéologue aient des ouvertures. On ne peut pas tout savoir, mais au moins avoir des ouvertures et comprendre, et pas inventer.

e-Phaïstos : De ce point de vue-là, il y a eu quelques travaux sur des paléopollutions liées à des ateliers disparus qui laissent des traces sur le très long terme parfois.

P.B. : Des travaux anglais sur les pollutions amenées par le travail du plomb dans des sables en contexte d'estuaires ou de baie remontent à au moins vingt ans si ce n'est plus. Il y a des pollutions comme celles que Marie-Christine Bailly-Maître a étudié à Brandes ${ }^{27}$ qui est une mine de plomb ; Florent Téreygeol ${ }^{28}$ a trouvé des sols très pollués aussi à Melle ${ }^{29}$, Nicolas Thomas ${ }^{30}$ a étudié aussi les pollutions, parce qu'il travaille sur des ateliers de production de cuivre, de bronze et de laiton, alors il y a du cuivre, mais il y a aussi du plomb du zinc et des tas de choses, même si pour ces productions c'est quasiment normal et on s'y attend, il a fait un travail remarquable. Ce qui est intéressant dans ce contexte c'est de voir les étendues de pollutions qu'ont pu susciter certains sites comme le site de Melle par exemple, ou les sites de production ou de traitement du plomb en Angleterre jusqu'au début de l'époque moderne : ce sont des grosses pollutions qui sont intéressantes aussi pour le monde actuel. C'est un problème qu'on avait vu aussi avec le Piren-Seine, pour des pollutions qui ne sont pas seulement des pollutions au premier degré : un curage de ruisseau, ça peut relancer un cycle de pollution, parfois après plusieurs centaines d'années.

e-Phaïstos : Tout ce qui était bloqué par les recouvrements colluviaux et alluviaux.

P.B. : C'est ça, le métal redeviendra minerai un jour, mais il faut lui laisser le temps et en l'occurrence l'archéologue (en fait tout mouvement du terrain) peut devenir un facteur de pollution.

e-Phaïstos : Pour en revenir à la circulation des matières premières, à l'époque médiévale, on fait circuler une partie du métal sous la forme de lingots, et vous disiez tout à l'heure qu'on avait en gros la même qualité de fer pour fabriquer des canons que des fers de construction.

PB. : Oui, hors la minette, on a en gros quatre types de fer (le fer lui-même reste le fer : Fe avec 
des tas d'inclusions) mais c'est son traitement et ce qu'il contient qui varie : lié au carbone, ça donne de l'acier; sa teneur en phosphore qui faisait hurler nos arrière-grands-pères, et nos grands-pères est jugée aujourd'hui comme relativement favorable car le phosphore permet d'abaisser le point de fusion et favorise la soudabilité, etc. Ainsi on peut caractériser l'état d'avancement d'un fer. Ce qui est ennuyeux pour un archéologue, c'est d'avoir un fer pur : on ne peut rien en dire, sauf qu'il est pur. Les fers qu'on a étudié à Fontenay, et ceux observés sur les cathédrales (ce n'est pas moi qui ai fait les analyses, mais j'ai lancé l'opération, c'est ce qu'a fait Maxime L'Héritier ${ }^{31}$ ), appartiennent à la même catégorie de fer, c'est-à-dire qu'ils sont un mélange de ferrite, de fer pur et d'acier. Ce sont des ensembles hétérogènes avec pas mal d'inclusions, alors les métallurgistes diront que c'est un fer qui est "sale », mais on le retrouve à peu près partout. C'est un niveau de traitement assez peu développé sur les grosses pièces, alors qu'on arrive à avoir sur des tranchants, sur des armes, même sur des armes mérovingiennes, en aciers beaucoup plus propres parce qu'il y a un énorme travail de forge. Cela explique aussi pourquoi les cathédrales consomment des quantités très importantes de fer, beaucoup plus importantes que nécessaire si on avait eu alors les aciers de notre époque : on a tendance à surdimensionner les armatures parce qu'on n'est pas tout-à-fait sûr de soi. On arrive comme ça à appréhender le niveau technique. Mais ce n'est pas seulement le niveau technique, et le niveau de la technique utilisée, on sait dès le haut Moyen Age et même avant faire d'excellents aciers, en particulier les barbares avaient des armes extraordinaires avec d'excellents aciers très épurés avec des teneurs en carbone tout-à fait convenables. On a des besoins économiques aussi : faire des aciers de bonne qualité, très épurés, coûte beaucoup plus que sortir des lingots et les transformer en barres. On a donc un type de fer que le professeur Gérard Bérenger, professeur de métallurgie à l'Université de technologie de Compiègne qualifiait « de fer à ferrer les ânes »; on retrouve le même d'ailleurs dans les fers à cheval.

e-Phaïstos : Cela est-il significatif de l'homogénéité des chaînes techniques et des chaînes opératoires?

P.B. : Oui et non. Je m'explique : oui, on a une certaine homogénéité des chaînes opératoires mais non parce que les appareils qu'on utilise sont parfois assez différents. On a des fers qui semblent de même qualité, avec des types de fourneaux de réduction qui ne sont pas les mêmes, mais malgré tout on a encore des lacunes en cette matière. Le résultat pour nous est le même parce que des éléments nous échappent complètement, c'est pour ça que le travail de Marc Leroy est très intéressant : il a pu expérimenter avec un type de four qui était reconstitué d'après ses fouilles et avec le même minerai que celui utilisé par les hommes de l'époque médiévale (du moins à très peu de choses près, à l'époque médiévale il était un tout petit peu plus riche en fer, mais ça compte pas car c'est le même minerai dont la partie la plus riche a été utilisée à l'époque médiévale). Il a donc pu travailler sur la quantité de charbon de bois utilisée, parce que le type de four a pu influer considérablement sur la consommation, même si le défaut d'expérimentation laisse encore la place au doute, il semble que plus le four est ouvert, plus il consomme. Il faut qu'il y ait un bon tirage quand même pour le faire chauffer le moins cher possible (le point d'économie est au maximum de chauffe avec le minimum de combustible), alors on patauge encore un peu parce que la technique ce n'est pas seulement le résultat, c'est aussi ce que ça coûte et le coût du minerai, à partir du moment où on peut le calculer, c'est-à-dire le début de l'époque moderne, est très inférieur à celui du combustible. Ça peut paraître difficile de transposer ça au Moyen Age parce qu'il y avait alors plus de plomb, comme on en consommait moins. Il ne faut pas oublier que la sidérurgie indirecte, grosse consommatrice de bois, a démarré au moment de la reprise économique et la reprise de l'ac- 
tivité agricole vers 1450 , à un moment où on avait du bois en abondance qu'on a coupé parce qu'il avait envahi beaucoup de terres qui n'avaient pas été cultivées pendant un siècle. C'est un des facteurs de développement, alors que la sidérurgie indirecte était déjà connue, elle marchait très bien en Suède où on estime qu'il y avait 5000 arbres pour un habitant.

e-Phaïstos : Ça me fait penser à une anecdote que m'a raconté un collègue : ils faisaient, voilà quelques années, avec son équipe un four expérimental pour tester le marquage du charbon de bois sur l'acier et c'est là qu'ils se sont rendus compte que la majorité des charbons de bois actuellement disponibles, sont en fait produits au pétrole et que ça pollue complètement le fer... Ils avaient des moyens pour acheter du matériel et faire des analyses, et c'est sur les analyses qu'ils ont monté leur protocole expérimental, sans envisager cette éventualité.

P.B. : [Rires] si on veut expérimenter sérieusement on fait son propre charbon de bois, c'est ce qu'on a fait à Fontenay, une fois qu'on a vu ce que ça a donné, on a pu l'acheter. Maintenant si on fait $\mathrm{du}$ fer pour tester notre marteau hydraulique, on achète le charbon de bois.

e-Phaïstos : Mais faire une meule à charbon c'est un très gros travail (en temps et en protocole expérimental), ça ne se fait pas en trois jours.

P.B. : En effet et c'est intéressant, c'est passionnant. On se rend compte, et c'est un autre aspect de l'archéologie, qu'on aura beau lire tout ce qu'on voudra, toutes les descriptions ethnographiques, les récits, les romans qui parlent de charbonniers, tout ce que les ethnologues racontent, si on n'a pas fait de charbon de bois, on n'imagine pas la difficulté, la technicité qu'il faut avoir. On a travaillé avec un charbonnier qui produit encore, c'est un bûcheron qui charbonne et les gens qui veulent du charbon bio pour leur barbecue vont le voir. Si ça peut mar- quer l'acier, ça doit pouvoir aussi marquer l'estomac...

e-Phaïstos : Qu'est-ce qu'on peut établir comme rapports entre histoire des sciences et histoire des techniques, où sont les points de contact : à quel moment on s'aperçoit notamment, ou est-ce qu'on ne le voit pas du tout d'ailleurs, qu'il y a une approche de type scientifique qui intervient dans la technique?

P.B. Ce n'est pas si simple que ça. Moi, je parle de moi, j'ai commencé par faire de l'histoire des sciences, puis j'ai plongé le nez dans la technique et j'ai refait de l'histoire des sciences. Ce qui m'a intéressé c'est les aspects pratiques des sciences : j'ai travaillé sur la mathématique des marchands. Ceci étant dit, je me suis aussi intéressé à l'histoire de la géologie au travers d'Agricola, je me suis rendu compte que les techniciens ont fait faire un grand bond en avant à certains aspects de la géologie, en particulier le père de la minéralogie qu'est pour moi Agricola. C'était un médecin, un homme politique (il était maire de son village) ; il écrivait énormément, il avait une puissance de travail colossale et il s'est intéressé à tout ce qui est souterrain, parce qu'il était médecin des mineurs. Il avait des intérêts dans les mines. C'est donc comme ça qu'il a ramassé tout un savoir qu'il n'a pas créé. Ramasser un savoir, c'est déjà très important, et ce savoir il l'a transformé en une science. Il y a science parce qu'il $\mathrm{y}$ a classement ; à partir du moment où on classe, on a une démarche scientifique. Pour moi Agricola est un technologue. Et c'est valable aussi en gîtologie : c'est le premier à avoir une vision un peu scientifique des sites minéraux. Sa représentation des gîtes minéralogiques est encore reprise dans l'Encyclopédie au XVIII ${ }^{\mathrm{e}}$ siècle ; plus exactement la représentation gitologique de l'Encyclopédie est encore héritée de celle d'Agricola et pourtant l'écart est important. Il y a donc là un rapport science et technique qui est intéressant, avec un apport des techniciens : des gens qui maitrisent la technique, mais qui deviennent capables d'élaborer un discours, alors que 
souvent le technicien a bien les idées dans la tête mais n'élabore pas un discours.

Je travaille actuellement sur un article qui développe la démarche inverse : à partir d'Agricola. C'est l'utilisation de la trigonométrie par les mineurs pour la topographie souterraine: on se situe aux confins du sud des pays germaniques et de la Tchéquie. C'est aussi là que sont apparus les premiers grands traités de la trigonométrie européenne : les Indiens puis les Arabes les ont transmis, et deux allemands, Puerbach et Regiomontanus ${ }^{32}$, l'ont redéveloppée. Cette trigonométrie va donner naissance à un appareil qui est une sorte de table sur laquelle on a matérialisé des sinus, avec un demicercle gradué et une aiguille, on reporte les graduations sur une règle et ça donne le sinus qu'on divise ensuite par le côté opposé, et on trouve le côté qu'on cherche. Alors, comment s'est fait ce passage ? C'est là-dessus que je cale un petit peu en ce moment. Ca s'est fait par des individus qui étaient à la fois intéressés par la mine et qui étaient en même temps des savants. Intéressés par la mine, pourquoi ? Agricola y avait des intérêts intellectuels, parce que l'homme est curieux et heureusement, mais aussi des intérêts économiques. Il y a donc là des rapports sciencetechnique qui m'intéressent beaucoup, que je vois au XVI ${ }^{e}$ siècle, mais sur lesquels il y a des gens qui sont sûrement beaucoup plus compétents que moi.

e-Pahïstos : Vous avez parlé d'Agricola technologue, avec une démarche scientifique. Comment faites-vous la distinction entre technique et technologie et comment définissez-vous cette dernière ?

P. B : Alors ça c'est surtout un problème de vocabulaire, les archéologues très souvent confondent les deux. Pour moi le terme de technologie est un mot du français qui est passé dans le monde anglosaxon, c'est un terme international puisque c'est du grec: techné et logos, mais il est passé dans le monde anglo-saxon où il est employé pour signifier technique, même technique de pointe, mais enfin j'ai publié un article dans History and Technology sous le titre « Technology and crisis... »33 qui est tout à fait convenable pour un Américain mais que je ne voudrais pas voir traduit par « Technologie et crise », parce que pour moi, et je ne suis pas le seul à avoir travaillé là-dessus (l'équipe de Lilianne Hilaire Perez a fait un séminaire sur la technologie et ce qu'elle doit être), la technologie commence avec la construction d'un discours logique, cohérent, je dirais même scientifique, sur la technique. C'est pour ça que je pense qu'on peut considérer Agricola comme un technologue : à partir du moment où il a classé les approches, il a classé les machines, il a classé les types de mines, etc. On rentre là dans une technologie et je retrouve un peu l'idée de Bertrand Gille qui a fait deux ouvrages (il en a préparé un troisième qu'il n'a pas eu le temps d'achever), le premier s'appelle Les mécaniciens grecs ${ }^{34}$, qui parle uniquement des traités sur la technique, le second c'est Les ingénieurs de la Renaissance 35 et porte aussi sur des traités, sur la vision qu'on en a. Pour moi, là on est dans la technologie. Forger une épée mérovingienne avec de très grandes qualités techniques, ce n'est pas de la technologie, c'est une technique, une technique de pointe ; c'est là que j'établis une différence. Alors évidemment avec ces deux visions, l'américaine et puis la nôtre, beaucoup de gens actuellement utilisent «technologie » au sens où l'entendent les Américains, et en particulier pour tout ce qui est technique de pointe, ils emploient pour les techniques actuelles le terme de technologie qui est en français un contre-sens, mais qui à mon avis finira par l'emporter parce que l'usage du français tend à s'effondrer devant les américanismes. Je pense au remplacement de par par by dans de très nombreuses publicités, et la publicité est un vecteur de transformation, en particulier du langage ; ce terme de technologie y revient sans cesse. Quand on voit des publicités à la télévision pour des voitures, c'est toujours «technologie » qui est utilisé : « les technologies de pointe », « les technologies les plus innovantes » etc...

Dans le rapport science et technique, ce qu'on peut dire aussi c'est que ce qui m'a fait goûter aux deux, ce n'est pas seulement des questions d'oppor- 
tunité de temps, c'est un goût très particulier pour ce qui me semble être les grands éléments qui ont fait les mutations de notre monde: mutations scientifiques, mutations techniques. Et c'est pour ça que le mémoire que j'ai fait pour ma thèse portait justement ce terme: Les mutations techniques et scientifiques de la fin du Moyen Age et de la Renaissance, si mes souvenirs sont exacts ${ }^{6}$ : le problème de mutation du monde à certaines époques qui sont à la fois scientifiques et techniques, alors maintenant évidemment tout ça c'est dans le même panier, c'est la «big science » comme ils disent maintenant.

e-Phaïstos : Ce qui est intéressant c'est qu'il n'y a pas de bascule brusque, ce sont vraiment des passages qui se font lentement.

P.B. : Oui il n'y a pas de passage brusque, enfin, si on prend la mécanisation comme exemple, ça va quand même très très vite.

e-Phaïstos : Oui mais parce qu'il y a des phases de progrès?

P.B. : Oui mais quand est-ce qu'elle commence cette phase de progrès ? Pour la sidérurgie, ça commence au moins au XII ${ }^{e}$ siècle, mais depuis deux mille ans, trois mille ans même qu'on sait faire du fer au Moyen Orient, en Afrique vers 2000 avant J.-C., ça fait de nous des retardataires, vers 1000 avant J.-C. On n'utilise la force hydraulique qu'à partir du XIIe siècle, même si on sait déjà faire de l'acier. Du XII ${ }^{\mathrm{e}}$ au XIX ${ }^{\mathrm{e}}$ siècle, on va de plus en plus vite et ça plus ça, on passe au marteau hydraulique, ensuite à la fonte, puis à la fonte au coke et puis au procédé Bessemer37, etc... La notion d'accélération, moi je la vois dans le temps long, l'histoire du monde ne commence pas au Moyen Age.

e-Phaïstos : Bien sûr, ce que je voulais dire c'est que le progrès technique finalement c'est quelque chose de continu, à partir du moment où on investit un champs technique, le progrès est continu, certes mais avec des moments d'accélération.

P.B. : Pour passer du choping tool, du galet aménagé, ne serait-ce qu'à la pointe de flèche moustérienne, il y a déjà un pas énorme, ce pas a duré plusieurs millénaires. Ce n'est pas nécessairement une évolution, mais une accélération du progrès technique. Il faut quand même relativiser si ça va très vite, il y a d'autres accélérations qui paraissent très lentes par rapport au monde actuel : j'avais étudié la démographie du bassin parisien avec les gens du Piren-Seine, qui ne voyaient que la montée en flèche aux XIX ${ }^{\mathrm{e}}$ et $\mathrm{XX}^{\mathrm{e}}$ siècles, je leur ai fait remarquer que la croissance de Paris, si on ne se place pas en terme de croissance, en pourcentage, pas en chiffres absolus, a été aussi importante au XIII siècle qu'au $\mathrm{XX}^{\mathrm{e}}$ siècle. Si on fait une courbe avec une échelle logarithmique, elle est continue dans les deux cas, si on fait une courbe avec les valeurs arithmétiques, on ne mesure plus la croissance mais la quantité.

Il y a donc des époques de mutation, pour moi la fin du Moyen Age est une grosse époque de mutation comme il y en a eu aux XII - -XIII ${ }^{\mathrm{e}}$ siècles, bien sûr à la fois intellectuelle, technique et économique, stoppée par la crise puis c'est reparti. L'époque moderne, dans le domaine technique, le XVII ${ }^{e}$ siècle en particulier est plutôt plat, mais au point de vue de la technologie, il se passe des choses parce qu'on réfléchit et même beaucoup et on aboutit au temps de l'Encyclopédie au XVIII ${ }^{\mathrm{e}}$ siècle, après la technique redémarre. C'est-à-dire que les courbes technique et technologie ne sont pas forcément parallèles.

e-Phaïstos : Alors comment avec ça peut-on avoir une vision d'anthropologie historique : l'intégrer dans " l'histoire globale », dans la compréhension de l'ensemble d'une société, pour le Moyen Age par exemple ? Parce que finalement, qu'on étudie la technique, le fait religieux ou autre chose, on est toujours dans une portion de la société.

P.B. : Une réponse globale ou une réponse par petits morceaux ?... En fait, la réponse globale per- 
sonne ne l'a. Mais ce que je peux dire, c'est que je ressens évidemment des liens extrêmement étroits entre tout ce qui est technique et économie, c'est évident. Et comme je crois que l'économie pèse très lourd dans tout ce qui est société, social « sociétal » on rentre dans l'économique et le social : les liens sont extrêmement étroits entre histoire économique et sociale et histoire des techniques. Quand on regarde la grande crise de la fin du Moyen Age, ou la crise de 1929, on se rend compte qu'à ces momentslà, pourtant ça paraît paradoxal, la crise va libérer. Libérer des énergies et libérer des techniques nouvelles, donner des forces à de nouvelles concentrations industrielles qui ne sont pas toujours profitables par ailleurs, mais c'est comme ça. Donc des liens extrêmement étroits. Avec la vie intellectuelle, c'est plus compliqué, on l'a vu avec l'histoire des sciences, il y a des liens, les liens avec la vie artistique sont évidents : sans ferraillage, il n'y a pas de cathédrales gothiques. J'ai fait un article làdedans ${ }^{38}$ sur le plomb à la cathédrale de Chartres, c'est très technico-économique, mais j'arrive à ces conclusions que le plomb ne coûte pas cher et que c'est de très loin le métal le plus utilisé dans une cathédrale (des centaines de tonnes surtout pour la couverture) et pourquoi a-t-on pu faire ça ? Parce que du plomb on en avait énormément et pas cher. Il s'agit d'un sous-produit du travail du minerai de plomb argentifère, il est donc abondant. Mais ce n'est pas le plomb qui fait le toit quand même, parce qu'il y a des endroits où c'était couvert en tuiles, et il ne tiendra pas tout seul. Dès qu'on sort de l'économique et du social, si on veut créer une sorte de modèle à partir de l'histoire des techniques, on déborde sur l'économique et le social et de là on va pouvoir rentrer, à partir du social surtout, dans les mentalités et les représentations. Mais on n'a pas de lien direct.

Une vision globale de l'histoire est toujours terriblement sujette à caution, si on prend quelqu'un comme Georges Duby, qui était un grand esprit, il donne des aspects qu'il veut générateurs d'idées le plus possible, mais il ne donne pas une explication du Moyen Age. C'est la part de la complexité de la société. Ce qu'on peut, c'est établir des liens et partir dans différentes directions, moi je pars des techniques et on établit des ponts et d'autres ponts peuvent s'établir après. On peut refaire à mon avis un schéma du même type en partant du politique par exemple et en passant par l'histoire des techniques ou en y aboutissant avec des ponts qui restent des passerelles un peu fragiles...

Comme disait Jean Favier : "on m'a traité d'arriviste, mais ne sont pas arrivistes ceux qui sont arrivés ». toire.

e-Phaïstos : Bref on est loin de la fin de l'his-

P.B. : Oui, il faut lire Asimov $39 . .$.

${ }^{1}$ A l'origine, il s'est surtout agit d'une dynamique d'équipe autour
d'une thématique mine et métallurgie qui s'est ensuite élargie vers une approche plus large d'histoire des techniques.

2 Antoine Prost intervenait en tant que président du conseil scientifique de la mission du centenaire de la Grande Guerre lors de la soirée de présentation du film Apocalypse - La première guerre mondiale, le 1e avril 2014 sur France 2.

3 Voir notamment: Yves Desfossés, Alain Jacques et Gilles Prilaux, L'archéologie de la Grande Guerre, Inrap-éditions ouest-France, 2008, 128 p. ; Collectif sous la direction de Bernadette Schnitzler et Michaël Landolt, A l'est, du nouveau! Catalogue d'exposition, 304 pages ; «Les traces archéologiques de la nourriture des combattants du front de Champagne (19151918) », Franck Lesjean, dans Etudes Marnaises SACSAM, Tome CXXVI - année 2011, p. 179-284. L'archéologie contemporaine se développe et donne lieu à un nombre croissant de publications.

4 Directeur de recherche, LA3M, UMR 7298, Université d'AixMarseille.

5 Marc Leroy Laboratoire de Métallurgies et Cultures - IRAMAT - UMR 5060 - Belfort-UTBM, Nancy-Musée de l'Histoire du Fer.

Leroy Marc, Merluzzo Paul, Le Carlier Cécile, Archéologie du fer en Lorraine. Minette et production du fer en bas fourneaux dans l'Antiquité et au Moyen Age. Fensch-Vallée éditions, Knutange, à paraître en 2014 ou 2015 (environ 375 p.). 
6 Ingénieur britannique qui met au point le procédé de déphosphoration des fontes issues de minerais phosphoreux pour les convertir en acier, en 1877-1879.

7 Association pour les Fouilles Archéologiques Nationales, association para publique dont l'objectif était de soutenir les fouilles archéologiques de sauvetage, créée en 1973, cette association s'est peu à peu renforcée pour devenir à la fin des années 1990 une véritable structure professionnelle, elle a été dissoute en 2001 lors de la promulgation de la loi sur l'archéologie préventive instituant l'INRAP (Institut National de Recherches Archéologiques Préventives), établissement public administratif sous la double tutelle du ministère de la culture et de la communication et du ministère de la recherche et de l'enseignement supérieur. Aujourd'hui, il existe plusieurs opérateurs d'archéologie préventive agréés pour les fouilles: L'INRAP, établissement public national, les services archéologiques de collectivités territoriales (actuellement 70 agréés, sur 103 services à l'échelle nationale source ANACT) et des sociétés privées (Evéha, Antéa, Hades, Arkémine, archéopole, archéodunum - liste non exhaustive)...

8 Les fouilles programmées sont réalisées sur la base d'un programme scientifique généralement pluriannuel et visent à répondre à des questions précises, elles sont généralement réalisées à l'occasion de campagnes annuelles avec des budgets clos ; les fouilles préventives sont réalisées en préalable aux travaux d'aménagement du territoire, à la suite d'un diagnostic visant à évaluer le potentiel archéologique des terrains. Les moyens de chaque fouille sont négociés entre l'opérateur d'archéologie préventive et l'aménageur en fonction d'un cahier des charges rédigé par les services régionaux de l'archéologie relevant de la DRAC (services déconcentrés du ministère de la culture) qui assurent le contrôle scientifique.

9 Les fouilles de la LGV Bretagne-Pays de Loire, portion RennesLe Mans ont permis dans le département de la Sarthe la découverte et la fouille de plusieurs sites métallurgiques (mines, ateliers de réduction, ateliers de forge et de préparation de l'acier) de l'Age du Fer au bas Moyen Age, sur les communes de Neuville-sur-Sarthe, Aigué, La Milesse, Degré ; http://www.inrap.fr/atlas/lgv-bretagne/sites-archeologiqueslgv-rennes-le-mans\#.U6dcIoAxjDM

10 Sprandel Rolf. «La production du fer au Moyen Âge. » In: Annales. Économies, Sociétés, Civilisations. 24e année, N. 2, 1969. pp. 305-321.; doi : 10.3406/ahess.1969.422055. http://www.persee.fr/web/revues/home/prescript/article/ahess _0395-2649_1969_num_24_2_422055

11 Jura, fouilles de la LGV Dijon-Mulhouse, découverte en 2012 d'un moulin à eau médiéval (1130-116o d'après la dendrochronologie - dates d'abattage) qui pourrait avoir été une dépendance de l'abbaye cistercienne de Thervay ; R.O. Gilles Rollier
12 La métallurgie par réduction directe à l'est de la Seine-Maritime Études pluridisciplinaires sur la production du fer de la Protohistoire à la fin du Moyen Âge, Thèse préparée sous la direction du professeur Anne-Marie Flambard-Héricher, 2013. Christophe Colliou est archéologue paléométallurgiste, chercheur de la société Arkémine.

13 Philippe Nowacki-Breczewski + était responsable du programme archéologique de Petit-Saut en Guyane, il est décédé en 1993.

14 Benoit P., Dormoy C., Lichon A.A. 1998 : « Le vivier de Fontmorigny : pêcheries et conservation des poissons, une approche archéologique », in L'ordre cistercien et le Berry, Actes du colloque de Bourges, 15-16 mai 1998, Bourges, Société d'achéologie et d'histoire du Berry Cahiers d'archéologie et d'histoire du Berry, 136, p. 149-157.

15 INRAP, ingénieur d'étude et de recherche, LAMOP UMR8589.

16 Medieval Copper, Bronze and Brass - Dinant-Namur 2014 History, archaeology and archaeometry of the production of brass, bronze and other copper alloy objects in medieval Europe (12th-16th centuries) 15,16 et 17 Mai 2014 - Dinant et Namur (Belgium). Colloque international organisé par le service public de Wallonie (SPW), l'institut National de recherches archéologiques préventives (INRAP), la Maison du patrimoine médiéval mosan (MPMM) et le Centre culturel régional de Dinant (CCRD).

17 les référentiels doivent encore être complétés.

18 Pour ne prendre qu'une référence: Cyril Stanley Smith. $A$ History of Metallography (The development of ideas on the structure of metals before 1890). The University of Chicago Press, 1960. In- $8^{\circ}, 291$ pages, 110 illustrations.

19 On connait le travail d'E. Leroy-Ladurie (Histoire du climat depuis l'an mil, Flammarion, Paris, 1983, puis Histoire humaine et comparée du climat, canicules et glaciers XIIIe-XVIIIe siècles, Arthème Fayard, Paris, 2004) et des gens qui travaillent autour de lui sur la date du ban des vendanges, il y a aussi ce qu'a fait Pierre Alexandre (Le climat en Europe au Moyen Age, contribution à l'histoire des variations climatiques de 1000 à 1245 d'après les sources narratives de l'Europa occidentale, $828 \mathrm{p}$. Paris éditions de l'EHESS, Paris 1987) qui a noté toutes les chroniques.

20 parmi les données archéologique les plus spectaculaires de la Bassée au haut Moyen Age, on signalera la « pirogue » de Noyensur-Seine, qui constitue également une documentation de premier ordre pour l'histoire des techniques : Mordant Daniel. « La barque monoxyle carolingienne de Noyen-sur-Seine (Seine-etMarne) ». In: Archaeonautica, 14, 1998. Construction navale maritime et fluviale. Approches archéologique, historique et 
ethnologique. pp. 23-27.

http://www.persee.fr/web/revues/home/prescript/article/nauti _0154-1854_1998_act_14_1_1182.

$21 \mathrm{http}: / /$ lamop.univ-paris1.fr/

22 UTC (Université de Technologie de Compiègne) créée en 1972, membre de l'alliance Sorbonne Universités, établissement universitaire et école d'ingénieurs, qui a développé un axe de recherche interaction homme-systèmes technologiques et sociaux au sein duquel la paléométallurgie a sa place.

23 UTBM (Université de Technologie de Belfort-Montbéliard) directeur du laboratoire Métalluriges et Cultures, UMR 5060.

24 Directeur de recherches, CNRS, CEA, IRAMAT, UMR5060.

25 CEA LAPA (Laboratoire archéomatériaux et prévision de l'altération), UMR3299, Sylvain Bauvais est protohistorien.

26 Maître de conférences à l'Université Paris 1 UFRo3, UMR 8589 LAMOP. Site du moulin Glinet, commune de Compainville, Seine-Maritime http://moulindg76.free.fr/sur_internet_o3o.htm.

27 Village minier médiéval de Brandes-en-Oisans (Isère - Commune d'Huez) http://www.iserepatri-

moine.fr/TPL CODE/TPL_SITEPATRIMONIAL/PAR TPL ID

ENTIFIANT/27/1272-base.htm

28 CNRS - Iramat

29 A Melle (Deux-Sèvres), ce sont des mines d'argent du haut Moyen Age qui ont été étudiées et fouillées ; http://www.minesargent.com/ la commune a mis en place suite à ces fouilles une plate-forme d'archéologie expérimentale des Arts du Feu

30 INRAP - Lamop

31 Maître de conférence à l'Université Paris 8, EA 1571, pouvoirs, savoirs et sociétés, UMR 5060, Institut de recherches sur les archéomatériaux.

32 Georg von Puerbach $(1423$ - 1461) et Johannes Müller von Königsberg dit Regiomontanus (1436 - 1476), élève du premier sont parmi les premiers européens à redécouvrir la trigonométrie et à en chercher les applications...

33 « Technology and crisis : The great depression of the Middle Ages and the technology of Renaissance (XIVth to XVIth centuries) », dans History and Technology, 1984, vol.1, 319-333.

34 Bertrand Gille, Les mécaniciens grecs, la naissance de la technologie, Paris, Seuil, 1980, 230 p.

35 Bertrand Gille, Les ingénieurs de la Renaissance, Paris, Hermann, 1964, $240 \mathrm{p}$.
36 Les mutations techniques et scientifiques de la fin du Moyen Age et de la Renaissance, sous la direction de Michel Serres, ANRT, Lille, 1993

37 Il s'agit d'un procédé industriel de fabrication de l'acier mis au point par l'Anglais Henry Bessemer (1813-1898) en 1855-1856 : la fonte en fusion est versée dans une cornue de métal (convertisseur) garnie d'un revêtement réfractaire, l'air insufflé sous pression par des orifices ménagés à la base de l'appareil provoque la combustion du carbone et des impuretés (http://www.universalis.fr/encyclopedie/procede-industriel-defabrication-de-l-acier/).

38 Chartres - Construire et restaurer la cathédrale (XIe-XXIe siècles), sous la direction d'Arnaud Timbert éditions septentrion, 2014

39 Une première trilogie Fondation (1951), Fondation et empire (1952) et Seconde Fondation(1953) est complétée par un second cycle : Fondation foudroyée (1982), Terre et Fondation (1986), Prélude à Fondation (1988) et L'Aude de Fondation (1993), tous traduits en français sont édités aux éditions Pocket et réédités chez Denoël. 